\title{
A note on maximal matchings in rooted products of paths and short cycles
}

\author{
Ivana Zubac \\ Faculty of Mechanical Engineering, Computing and Electrical Engineering, \\ University of Mostar \\ Matice hrvatske bb, BA-88000 Mostar, Bosnia and Herzegovina
}

\begin{abstract}
A matching $M$ in a graph $G$ is maximal if it cannot be extended to a larger matching in $G$. The enumerative properties of maximal matchings are much less known and researched than for maximum and perfect matchings. In this paper we present the recurrences and generating functions for the sequences enumerating maximal matchings in rooted products of paths and short cycles. We also analyze the asymptotic behavior of those sequences.
\end{abstract}

Keywords: maximal matching, rooted product of graphs

MSC: 05C30, 05C70, 05C76

\section{Introduction}

A matching in a graph is a collection of its edges such that no two edges in this collection have a vertex in common. Many problems in natural, technical and social sciences can be successfully modeled by matchings in graphs. Today the matching theory is a well developed part of graph theory, strongly influenced by chemical applications. Particularly well researched are perfect and maximum matchings. For a general background on matching theory and terminology we refer the reader to the classical monograph by Lóvasz and Plummer [4].

A matching is perfect if its edges are collectively incident to all vertices of $G$. It is clear that perfect matchings are as large as possible and that no other matching in $G$

DOI: https://doi.org/10.5592/CO/CCD.2018.13 
can be "larger" than a perfect one. In all other applications we are also interested mostly in large matchings. If we are using the number of edges as the measure of "largeness", we get the maximum matchings. For them we have well developed structural theory and many enumerative results. No such theory, however, exists for another class of large matchings - maximal matchings. A matching $M$ is maximal if it cannot be extended to a larger matching in $G$. Obviously, every maximum matching is also maximal, but the opposite is generally not true. Maximal matchings are much less researched, but still very useful. That goes both for their structural and their enumerative aspects. The crucial difference is non-locality. That means that we cannot split the set of all maximal matchings into those containing an edge $e$ and those not containing it without taking into account the edge-neighborhood of $e$. The main goal of this work is to increase our knowledge about the enumerative aspects of maximal matchings.

We begin by establishing the principal terminology and notation which we will use throughout the article. It is taken from [7]. All graphs $G$ considered in this paper will be finite and simple, with vertex set $V(G)$ and set of edges $E(G)$. We will denote by $n=|V(G)|$ the number of vertices and by $m=|E(G)|$ the number of edges in $G$. As usual, the cycle and complete graph on $n$ vertices are denoted by $C_{n}$ and $K_{n}$, respectively. However, by $P_{n}$ we denote the path of length $n$, i.e., on $n+1$ vertices. Let $\Psi(G)$ denote the number of maximal matchings in graph $G$.

\section{Rooted product of graphs}

Many interesting graphs arise from simpler building blocks via some binary operations known as graph products. In 1978, Godsil and McKay [2] introduced a new product of two graphs $G_{1}$ and $G_{2}$, called the rooted product, and denoted by $G_{1} \odot G_{2}$. The rooted product graphs are used, for example, in internet networking for connecting local networks to a wider frame. One can view such a situation as a combination of many local networks (copies of graph $H$ ) having a server (the root vertex of graph $H$ ). These servers are themselves connected through a global network (the graph $G$ ). So, one motivation to count the maximal matchings can be securing networks with as few sensors as possible.

The main goal of this paper is to study the enumerative aspects of maximal matchings in some classes of rooted products. We establish recurrences for the enumerating sequences and, in some cases, we use generating functions to determine 
their asymptotic behavior. We consider operations of rooted product of paths and cycles, and enumerate maximal matchings in the resulting graphs.

Let $V(G)=\left\{u_{i} \mid 1 \leq i \leq n\right\}$ and $v \in V(H)$. The rooted product $G \odot H$ of a graph $G$ and a rooted graph $H$ with respect to the root $v$ is defined as follows: take $n=|V(G)|$ copies of $H$, and for every vertex $u_{i}$ of $G$, identify $u_{i}$ with the root $v$ of the $i$-th copy of $H$. Obviously,

$$
\begin{aligned}
|V(G \odot H)| & =|V(G)||V(H)| \\
|E(G \odot H)| & =|E(G)|+|V(G)||E(H)| .
\end{aligned}
$$

Hence, we take as many copies of graph $H$ as we have vertices in graph $G$. If $H$ or $G$ is the singleton graph, then $G \odot H$ is equal to $G$ or $H$, respectively. In this sense, to obtain the rooted product $G \odot H$, hereafter we will only consider graphs $G$ and $H$ of orders greater than or equal to two. We note that the rooted product is not commutative.

The rooted product is especially relevant for trees, as a rooted product of two trees is another tree. For instance, [3] used rooted products to find graceful numberings for a wide family of trees. Also, the rooted product of two graphs is a subgraph of the Cartesian product of those two graphs.

\section{$3 \quad$ Rooted product graph $P_{n} \odot P_{m}$}

We first consider the case when we have a rooted product of two paths, always taking a leaf as a root. In all cases we obtain a tree. An example is shown in Figure 1 .

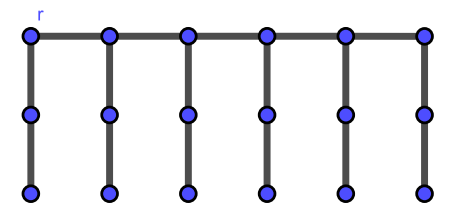

Figure 1: Example of $P_{n} \odot P_{2}$ rooted product

The next lemma is a direct consequence of Proposition 6.1. from article [1]. 
Lemma 1. The number of maximal matchings in rooted product graph $P_{n} \odot P_{1}$ is equal to the value of the $(n+2)$-nd Fibonacci number, i.e. $\Psi\left(P_{n} \odot P_{1}\right)=F_{n+2}$.

Proof. It is clear that every vertex of the $P_{n}$ must be covered by an edge of a maximal matching. If vertex $n$ is covered by the edge $v_{n-1} v_{n}$, the remaining edges of a maximal matching must form a valid maximal matching in $P_{n-2} \odot P_{1}$, and hence are counted by $\Psi\left(P_{n-2} \odot P_{1}\right)$. If $v_{n}$ is covered by the pendent edge of $P_{1}$, the remaining maximal matchings are counted by $\Psi\left(P_{n-1} \odot P_{1}\right)$. Hence, the number of maximal matchings in $P_{n} \odot P_{1}$ satisfies the recurrence

$$
\Psi\left(P_{n} \odot P_{1}\right)=\Psi\left(P_{n-1} \odot P_{1}\right)+\Psi\left(P_{n-2} \odot P_{1}\right),
$$

with initial conditions $\Psi\left(P_{0} \odot P_{1}\right)=1, \Psi\left(P_{1} \odot P_{1}\right)=2$.

The sequence $F_{n}$ of Fibonacci numbers is defined by the same recurrence relation and the same initial conditions, so the claim follows.

We now give another result for a rooted product of two paths, this time for the case $P_{n} \odot P_{2}$ shown in Figure 1. We will denote the number of maximal matchings in the graph $P_{n} \odot P_{2}$ with $t_{n}$.

Proposition 1. The sequence $t_{n}$ satisfies the recurrence $t_{n}=t_{n-1}+2 t_{n-2}+t_{n-3}$ with the initial conditions $t_{0}=2, t_{1}=4$.

In the proof we will repeat argument from paper [6] - we split graph into two parts: counting the number of matchings containing the root and counting the matchings which do not contain the root. We must take into account the edgeneighborhood of $e$.

Proof. Each maximal matching in $P_{n} \odot P_{2}$ either covers the rightmost root or does not cover it. In the first case, the remaining edges must form either a valid matching in $P_{n-1} \odot P_{2}$ (if is root covered by edge of $P_{2}$ ) or a valid maximal matching in $P_{n-2} \odot P_{2}$ (if the root is covered by edge of $P_{n}$ ).

Maximal matchings that do not cover that root must cover both its neighbors. The neighbor in $P_{n}$ can be covered in two ways, one of them counted by the number of maximal matchings in $P_{n-2} \odot P_{2}$, another by the number of maximal matchings in $P_{n-3} \odot P_{2}$. The claim now follows by adding the two contributions.

The following proposition and corollary give the generating function and asymptotic behavior of $t_{n}$. 
Proposition 2. The generating function $T(x)$ for the sequence $t_{n}$ is given by

$$
T(x)=\frac{x^{2}+2 x+2}{1-x-2 x^{2}-x^{3}} .
$$

Corollary 1. The asymptotic behavior of $t_{n}$ is given by $t_{n} \sim 1.92485 \cdot 0.51952^{n}$.

The sequence $t_{n}$ provides a new combinatorial interpretation of sequence $A 141015$ from the OEIS [5].

\section{Rooted product graph $P_{n} \odot C_{m}$}

In this section we will show some results about rooted product graph $P_{n} \odot C_{m}$. Figure 2 shows the first case - the rooted product $P_{n} \odot C_{3}$.

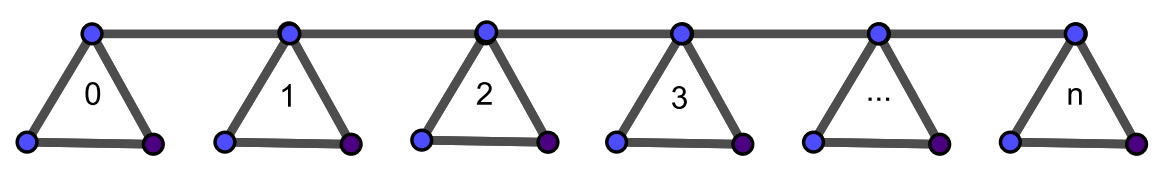

Figure 2: Example of $P_{n} \odot C_{3}$ rooted product

The next lemma is a consequence of Proposition 7.3. from article [1]. We will denote the number of maximal matchings in the rooted product graph $P_{n} \odot C_{3}$ with $p_{n}$.

Lemma 2. The sequence $p_{n}$ satisfies the recurrence

$$
p_{n}=2 p_{n-1}+3 p_{n-2}+p_{n-3}
$$

with the initial conditions $p_{0}=3, p_{1}=9, p_{2}=28$.

The sequence $p_{n}$ appears as $A 084084$ in the OEIS.

An example for the next case, the rooted product $P_{n} \odot C_{4}$, is shown in Figure 3 . We will denote the number of maximal matchings in this case with $\Psi\left(P_{n} \odot C_{4}\right)=r_{n}$. 
Proposition 3. The sequence $r_{n}$ satisfies the recurrence

$$
r_{n}=2 r_{n-1}+4 r_{n-2},
$$

with the initial conditions $r_{0}=2, r_{1}=8$.

Proof. Each maximal matching in $P_{n} \odot C_{4}$ must cover the root of the copy of $C_{4}$ rooted at the rightmost vertex of $P_{n}$. If the root is covered by one of the two edges of $C_{4}$ incident with it, the remaining edges of this maximal matching must form a valid maximal matching in $P_{n-1} \odot C_{4}$. If the root is covered by the edge incident with it in $P_{n}$, the remaining edges must form a valid maximal matching in $P_{n-2} \odot C_{4}$. In that case, there are four ways to form maximal matchings in the rest of the two rightmost copies of $C_{4}$. The claim now follows by adding the two contributions.

Proposition 4. The generating function $R(x)$ for the sequence $r_{n}$ is given by

$$
R(x)=\frac{1}{1-2 x-4 x^{2}} .
$$

Corollary 2. The asymptotic behavior of $r_{n}$ is given by $r_{n} \sim 1+\sqrt{5}$, twice the golden ratio.

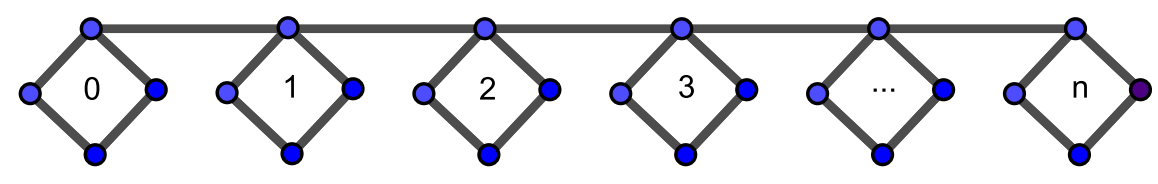

Figure 3: Example of $P_{n} \odot C_{4}$ rooted product

The sequence $r_{n}$ is known as the Horadam sequence $a_{n}=s \cdot a_{n-1}+r \cdot a_{n-2}$ for $s=2, r=4$. It appears as $A 085449$ in the OEIS and it counts, among other things, the number of ways to tile an $n$-board with two types of colored squares and four types of colored dominoes. 


\section{$5 \quad$ Concluding remarks}

In this paper we have counted maximal matchings in rooted products of some graphs. We have addressed only the combination of paths and short cycles. It would be probably too ambitious to hope for general enumerative results for rooted products. However, many interesting cases should be within the reach. Another interesting thing in such graphs would be to consider their saturation number and to compute their maximal matching polynomials.

\section{References}

[1] T. Došlić, I. Zubac, Counting maximal matchings in linear polymers, Ars Math. Contemp. 11 (2016) 255-276.

[2] C. D. Godsil., B. D. McKay, A new graph product and its spectrum, Bull. Aust. Math. Soc. 18 (1) (1978) 21-28.

[3] K. M. Koh, D. G. Rogers, T. Tan, Products of graceful trees, Discrete Math. 31 (3) (1980) 279-292.

[4] L. Lovasz, M. D. Plummer, Matching Theory, North-Holland, Amsterdam, 1986.

[5] N. J. A. Sloane, The On-Line Encyclopedia of Integer Sequences, Notices Amer. Math. Soc. 50/8 (2003) 912-915.

[6] S. G. Wagner, On the number of matchings of a tree, European J. Combin. 28 (2007), 1322-1330.

[7] D. B. West, Introduction to Graph Theory, Prentice Hall, Upper Saddle River, 1996. 
